\title{
Síndrome de Kartagener en el niño
}

\author{
Dres. Jaime Valenzuela B.*; Sylvia Medina P.**; Claudio Santander K.**; Alberto Gyhra S.***
}

\begin{abstract}
Three cases of Kurtakerser's Syrdrome diagmosed in the Pediatric Respiraton. Unit. Hospital Guillenno Grant Benavente, are analized. This rare syndroine was described for the first time in 1903.

Our cases a boy and two girls between six and eight veark of alse present an history of chronic bronchitis since the first months of year-life. Other causes of chronic bronchitis were discharged. Two of thein suffered also bronchial asma.

Bronchography denlostrated right un ilateral lasal bronkhiectusis irt two. and lett basal bronchiectasis in the third.

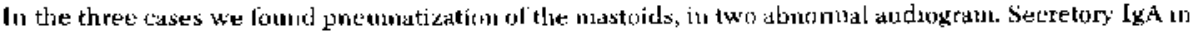
saliva was found low normal ul all them.
\end{abstract}

El Síndrome de Kartagener consistente en Situs Inversus, bronquiectasias y sinusitis cronica. Fue descrito por Siewert en 1904 y por Oeri en 1909; Kartagener en el año 1933, es el primero en plantear que esta asociación de patologías no es una simple coincidencia, presentando 4 casos ese mismo año y posteriormente 216 casos hasta $1956 .^{13}$

La incidencia de Situs inversus en material de necropsias, varía entre $1 \times 6000$ a $1 \times 10000$ y en ese material el Síndrome de Kartagener se encuentra con una frecuencia del 8 - $20 \%$. En nume. rosos estudios se han encontrado asociados al Situs inversus, malformaciones cardíacas, verte.

-Departamento de Pediatría. Universsdad de Concepxión y Servicio de Pediatria, Ilospital Gino. Grant Bellaveste. Concepcibin.

*"Servicio de Pediatria, Hospital Ginc. Grant Benavente. Concepción.

***Servicio de Cirugía, Hospital Gimu. Grant Benaverite. Consepción. Departamento de Cirugía, Universidad de Concepcion. brales y viscerales: pero, no se han encontrado Bronquiectasias en neomortinatos o en recién nacidos. ${ }^{1,13}$

En el Síndrome de Kartagener, la dextrocar. dia corresponde al tipo con imagen en espejo, la cual se asocia a malfonnaciones en una frecuencia similar al ate ofrece la población general. Los cambios electrocardiográlicos de esta dextrocardial son caracteristicos: onda P, QRS y onda T invertidas en primera derivacion.

En los últimos 20 años se han realizado numerosos estudios, tendientes a encontrar una relación en el mecanismo que conduce a la sinusitis crónica y a la bronqujectasia, llegándose a confirmar la hipótesis que el Síndrome de Kartagener tiene una determinación de tipo genético, manifestada en una alteración de la ultra estructura de los cilios del epitelio del árbol respiratorio, que determina un déliçit en el novimiento ciliar. El árbol tráqueo-bronquial cuenta con células que 
poseen 300 cilios, que laten a un ritmo de 1.300 movimientos por minuto. Esto da al manto mucoso una velocidad de desplazamiento $20-22$ milimetros por minuto. Cada cilio está forınado por 9 filamentos periféricos de doble estructura y 2 filamentos centrales. En el Sindrome de Kartagener existiria una alteración en los filamentos periféricos que carecerian de un tipo de las 3 variedades de conexiones que mantiener el conjunto de filamentos periféricos y central reunidos (Foto I.)

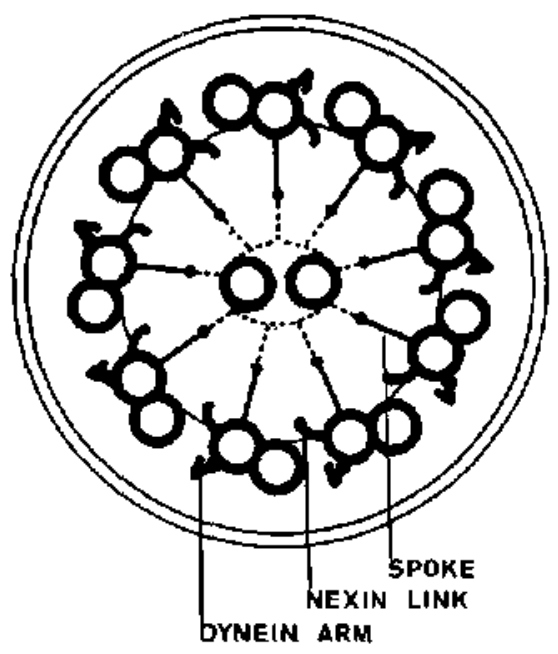

Figure 2. Diagram of the Cross-section of a Cillum or of the Central Portion of a Sperm Tall.

The aseembly of the nine outer microtubular doubiets and the two central microtubules is held together by three kinds of connections: the dyneln arms, the nexin links and the spoke. The dynetn arms are generaly betibred to be responslble for the motility.

\section{RTP. "THE TNGLAND JOURNAI MEDICINE" VOL * \& JULIO, $1977 \mathrm{~N}^{\circ} \mathrm{I} .-3$ RTEE ZLIASSON $\vee$ COLAB,-}

Foto 1

Elleason y colaboradores han demostrado que las pacientes portadores del Sindrome tienen un transporte inucociliar lento, que determina una marcada retención de secreciones, que fluctiian entre un $90-97 \%$. Los valores obtenidos con técnicas radioisotópicas en sujetos notmales son más bajos. ${ }^{4,3}$

Estudios efectuados en relación a la función auditiva, sugieren que en el Sindrome de Kartagener se presenta una escasa neumatizacón del apófisis mastoide con disminucíón de la función auditiva. Estos hallazgos podrian formar parte del Sindrome mismo, a sólo ser alteraciones secundarias a infecciones rinosinusales recurrentes. ${ }^{1 ; 3}$

\section{CASOS CLINICOS}

\section{CASOL}

Niña de 6 años I I meses, sin antex edentes familiares de importaltcia; acusa broncyutis obstructiva recidivante descle los 2 merses de edidl $x$ adernás, es portadora de rinitis crobencia.

La radirusralia de Thrax y E.C.G., revela situs inversos. Lat bronicografia mostra dilatación de los brompuion iontes]mondientes al seymento basal lateral del lobulo interor derecho (Foto 2 ).

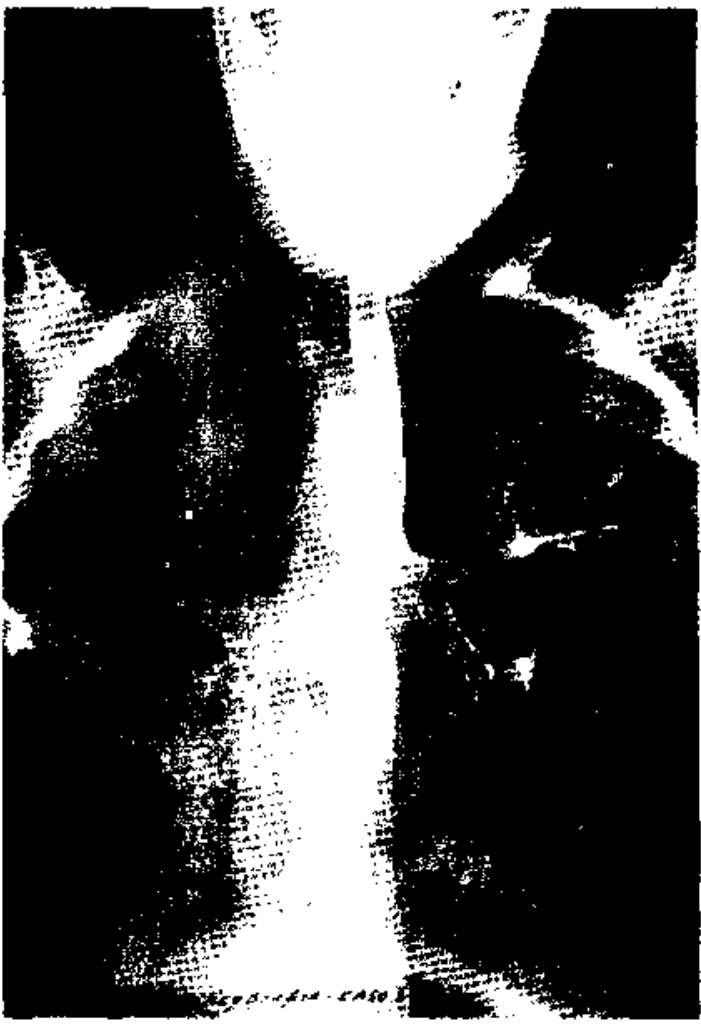

Folo2

Jit radioyrutia de senos paranasales, da velamiento de ambos senos maxilaxes, cun ansencia de desarrollo de senos frortaics. En la radiografia de Mastoides se encuentra ausencia de neumatizacion (Foto 3).

El diagnósticr del Síndrome fue efectuado a los 4 dû̀os.

\section{CASO2}

Niña de $\gamma$ años, sin antecedentes familiares, Bronquitis recurrentes desce el príner it)es de vida; portadora desde los 6 años de Rinitis Crónica. La radiografia de Tórax y E.C.C;, muestra Situs inversus total, con imagenes aerolares y cordonales en ambas bases, sugerentes de broncuiestasias. En la broncograffa hay dilatacioues bronquiales cilíndricas en el lobulo inferior 
derecho. Lat radiografia de senos palandsales, da vela-

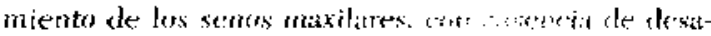
trollo de los senus frontatles i fuito 4 :

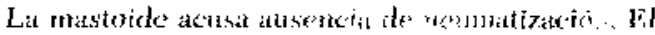

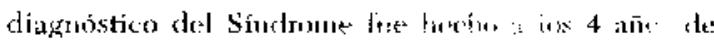
edad.

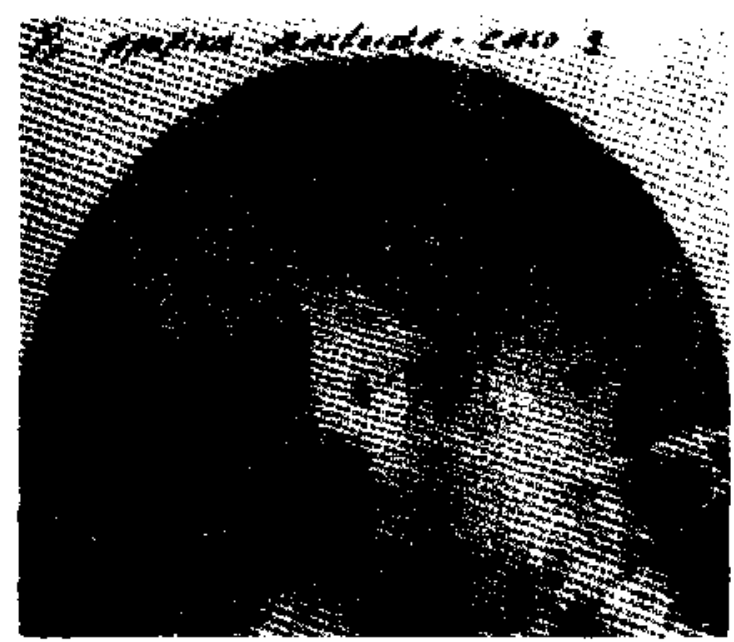

Fote 3

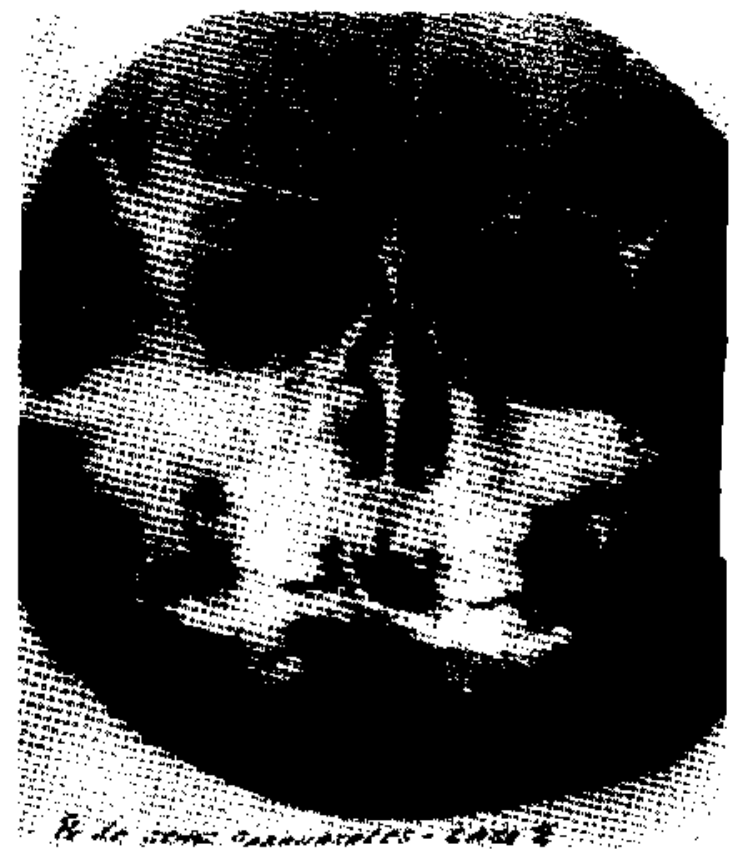

Foto 4

\section{CASO 3.}

Niño de $y$ años, vacurado BCG.Padre The en tratamienti). Bronguitis recurrette desde los 6 mekes de edad: bronconeumonia izequierda ell dos oportunidades. La radiografía de Tórax y E.C.G., revela Situs Inversus total, tracción de mediastino hacia la izquierda, con retracción de espacios intercostales y opacidad homoxénea de hemritórax izçuierder $\{F(y) 3 i$.

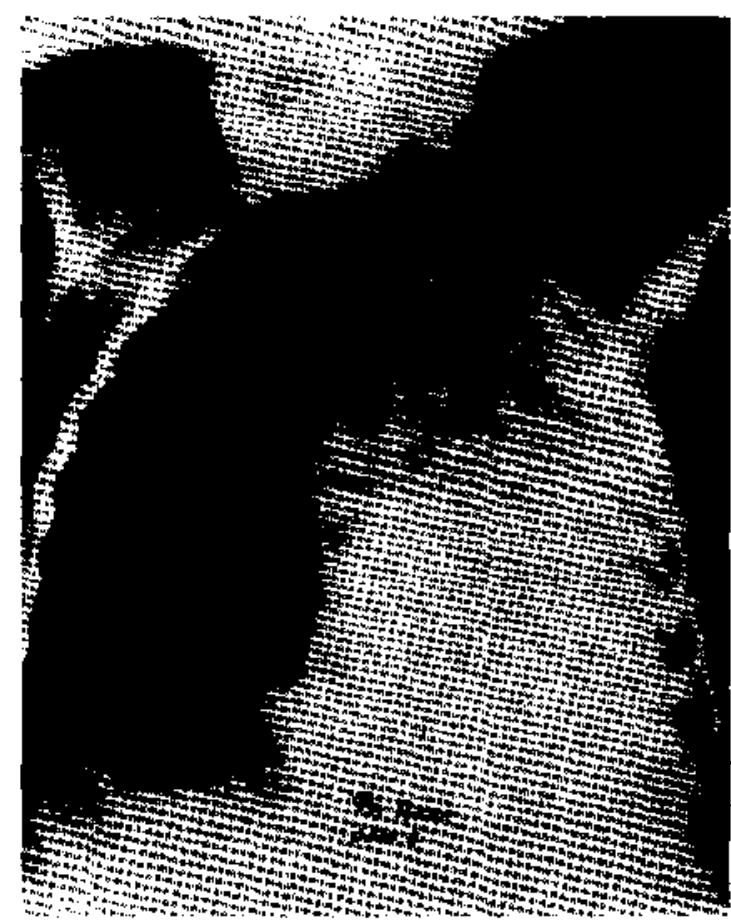

Folo 5

En la broncografía, dilataciones sacciformes del ibbulo superior izquierdo $y$ estenosis del bronquio inferior izquierdo, que deja pasar escaso medio de contraste. El diagnóstico de Sindrome fue hecho a $\operatorname{los} 3$ años de edad.

Velamiento radiográfico del seno maxilar izquier(do, atusencia de desarrollo de los senos trontales y ausencia de neumatización mastóidea.

Se realiza Neumonectomía Izquierda, evidenciánclose que el pulmón izquierdo anatómicamente correspondela al pulmón derecho. Su estudio histopatológico confirma bronquiectasias con fibrosis septo y Bronquitis crónica.

El estudio irmmunolígica en los tres nin̄os tnuestra Immunoglobulinas Séricas y Secretoras, dentro de los límites normales bajos.

Tabla 1

\begin{tabular}{|c|c|c|c|}
\hline & 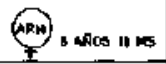 & (17) . NAlos & (100) O Afors \\
\hline RNDOPRAFIA TORAX & 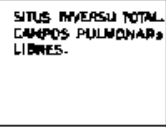 & 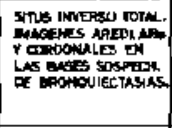 & 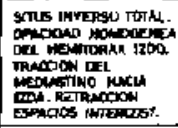 \\
\hline 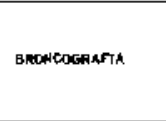 & 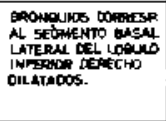 & 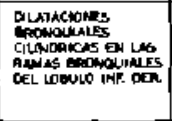 & 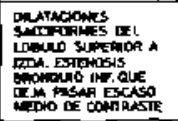 \\
\hline $\begin{array}{l}\text { RX BENGS } \\
\text { PAAMKASNIES }\end{array}$ & 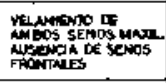 & 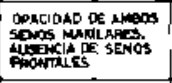 & 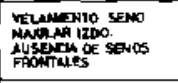 \\
\hline 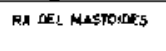 & 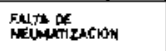 & 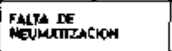 & PAITA EF \\
\hline
\end{tabular}


Tabla 2

ESTUDKO INMUNOALERBICO EN 3 CASOS CON SINDROME DE KARTAGENER

\begin{tabular}{|c|c|c|c|}
\hline 1.6A SECRETORIA & $1.5 \mathrm{mg} x$ & $13 \mathrm{mg} t$ & $9.5 \mathrm{mg} x$ \\
\hline $\begin{array}{l}\text { IWMUHOCLOBUUNAS } \\
\text { SERICLS }\end{array}$ & 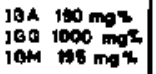 & 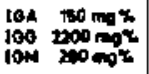 & $\begin{array}{l}I 04300 \mathrm{mg} \% \\
C_{3}+4 \mathrm{mg} \%\end{array}$ \\
\hline $\begin{array}{l}\text { TEST CATAHEO } \\
\text { ALERAICO }\end{array}$ & 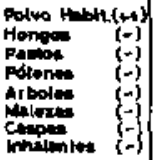 & 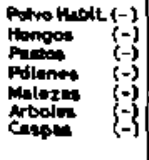 & 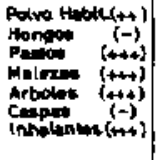 \\
\hline EOStMOFILIA NASAL & $50-75 \%$ & $10.25 \%$ & $20-50=$ \\
\hline 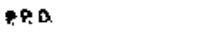 & $\{-\}$ & $\{-\}$ & $20 \mathrm{~mm}$ \\
\hline
\end{tabular}

\section{DISCUSION}

Los 3 niños motivo de esta comunicación, acusan desde los primeros meses de vida cuadros bronquiales a repetición, Rinitis crónica con descarga nasal purulenta. En ninguno de ellos existen antecedentes familiares de Situs Inversus y en su examen físico no presentan mal formaciones cardíacas, vertebrales ni viscerales, ajenas a los componentes dei sindrome. Los exámenes radiológicos realizados en todos ellos muestran Situs Inversus, Bronquiectasias, Sinusitis y ausencia de neumatización de la apófisis mastoides. En dos niños el test cutáneo de alergia, revela positividad asociadas a eosinofilia nasal sobre $\mathbf{2 5 \%}$, confirmatoria de Alergia Respiratoria. En el caso con antecedentes de contacto tuberculoso, se descarta enfermedad tuberculosa, mediante el estudio clínico bacterio-histopatológico.

Ha sido descrito como causal de infecciones respiratorias recurrentes, el déficit de IgA Secretoria, investigación que en todos nuestros pacientes da resultados en rangos normales. Puede atribuirse a la alteración mucociliar ya descrita asociada en dos casos a alergia respiratoria, la alta incidencia de cuadros respiratorios recurrentes que han presentado hasta la fecha. Por otra parte son estas infecciones recurrentes asociadas a Obstrucción Bronquial, las que generan las dilataciones bronquiales, por tanto es un hecho que las bronquiectasias en el Sindrome de Kartagener no son congénitas, sino que de aparición tardía y adquiridas. Esto se confinna por el hecho que no han sido descritas bronquiectasias en recién nacidos, con Situs Inversus.

Lo que da pues el sello al Síndrome de Karta- gener es la presencia simultánea de Situs Inversus y alteración del transporte mucociliar, determinada genéticamente. ${ }^{13,4,14}$

La neumatización de la apófisis mastoides se inicia al nacimiento y finaliza alrededor de los 3 años. En un $19,5 \%$ de la población, este proceso no se efectía lo que para algunos sería consecuencia de infecciones óticas y/o rinosinusales.

El rol patogénico de la inhibición de la neumatización, es discutible; talvez predispondría a inflamaciones, e infecciones del oido con tendencia a la cronicidad. Por otra parte, la falta de neumatización, puede reducir la capacidad auditiva por interferencia con las oscilaciones timpánicas. En nuestro material, se encontró en un caso disminución leve de la transmisión aérea (20 decibeles) y en otro una disminución importante de la misma (60 o más decibeles). Las referencias nacionajes de este Síndrome son escasas.

\section{RESUMEN}

Se analizan las fichas clinicas de 3 pacientes portadores de un Sindrome de Kartagener, controlados en el Consultorio Broncopulmonar del Servicio de Pediatría del Hospital Guillermo Grant Benavente de Concepción. Se analizan sus manifestaciones clínicas, radiológicas del Súndrome y se discute su etiopatogenia.

Los pacientes analizados corresponden a 1 varón y 2 niñas. Sus edades fluctúan entre los 6 y 8 años de edad. Todos con historia de bronquitis crónica desde los primeros meses de vida. La búsqueda de otras causas de Síndrome Bronquial Crónico, evidencia en dos pacientes Asma Bronquial Concomitante.

El estudio broncográfico demuestra bronquiectasias unilaterales, basales, en 2 casos a derecha y en el otro a izquierda. Falta de neumatización mastoídea y en todos audiometria alterada en dos. El estudio de IgA secretora en saliva, es normal en todos, en valores límites bajos.

\section{REFERENCIAS}

${ }^{1}$ E., Arge; N. B., Copenhagen. "Transposition of the viscera and sterility in Man". Lancet $1: 412,1960$.

${ }^{2}$ Commes, P.; Mosberg, A. A. F.; Meliws, B. "Evidence for congenitally nonfunctioning cilia in the tracheobronquial tact in two subjects. Am. Rev, Respir. Dis, 112: 807, 1975.

${ }^{3}$ Dalhamn, $T$,; Ever, G.: Fahlen, T. "Ciliary Beat rates in simus and bronchial mucosa from bronchiectatic persons". Arch Otolaryngol 70: 25, 1959.

${ }^{4}$ Elliason, $R$. et al. "A congenital giliary abnormalites as an etiological factor in chronic airway infection and male". N. Enkland J. Med. 297; 1, 1977? 
${ }^{5}$ Goldman, A.; Goldblum, R. "Primary deficiences in humoral inmunity". Pediatr. Clin. North Am. 24: 277, 1977.

Brant, R. P. "The Syndrome of dextoversion of the heart circulation". 18:25, 1958.

${ }^{7}$ Homwer, Blenerhosset, $J$. B. and Austen. "A reappraisal of Kirtiagener's Syndrome“ Am. J. Med. Sti. 225: 1.3, 1968.

Johanner, A, C.; Rhodin. "L']traestructure and function of the human tracheal mucosa". E. P. Radford "The Ciliated cell" Am. Rev. Respir. Lis. H3: 1.15 Tratado de Pediatria Part II, 1966.

${ }^{9}$ Moriber Katl. Sh. et al. Hannenian Medical College and Hospital "Kartagener's Syndrume and abnormal cilia". N. Engl. Med. 297: 1011, 1977.

${ }^{10}$ Nelson Vaughom Mc Koy. "Posiciones anómalas del corazón. Dextrocaardia y Levocurdia". Tono II 10089-6a. Ed, Salvat. 1971 .

${ }^{11}$ Richard, E.; Stielm, M. Dand Hug Fidenberg Md. "Senum levels of innunoylobuline in health and Disease. Pediatrics 37: 715,1966 .

12R. V'iedement. Harrburgo. "Significución fisiológiea de Apofisis Mastoides". Tratado de Otorinolaringología. J. Bhendes. R. Link Zolhmer (252-255), 1969.

${ }^{13}$ Sethei, $B$ r. "Kartagener's syndrome and its otological manifestations". J. Laryngol Otol. 99: 183, 1975.

147. J. Nexber et al. Nidle car ciliane detect in Kartagener's Syndrome". Pediatrics 62: 443, 1978.

${ }^{15}$ Dres. Garretón, U. 1.; Godoy, B. L. "Sindrome de Kartagener". Anales Medicos, Concepción 12: 33, 1955.

${ }^{16}$ Nazar, C.J. "Síndrome de Cartagener". Rev. Otorrinol. 30: $\$ 9$, 1970.

${ }^{17}$ Diqne, Wilson, Bichard Tálamo. "Aspectos genéticos de las neumopatias peduatricus". Pediatr. Clin, North Am. 3: 463, 1979. 